$\xi=-1$

\title{
Optimal Features Selection for X-Ray Image Retrieval with Regular and Irregular Zoning Methods
}

\author{
P. Nalini ${ }^{1 *}$, Dr. B. L. Malleswari ${ }^{2}$ \\ IDepartment of Electronics and Communications Engineering, MGIT, Hyderabad \\ 2 Department of Electronics and Communications Engineering, SWEC, Hyderabad \\ *Corresponding author E-mail pasumarthinalini@gmail.com
}

\begin{abstract}
Medical Image Retrieval is mainly meant for enhancing the healthcare system by coordinating physicians and interact with computing machines. This helps the doctors and radiologists in understanding the case and leads to automatic medical image annotation process. The choice of image attributes have crucial role in retrieving similar looking images of various anatomic regions. In this paper we presented an empirical analysis of an X-Ray image retrieval system with intensity, statistical features, DFT and DWT transformed coefficients and Eigen values using Singular Valued Decomposition techniques as parameters. We computed these features by dividing the images in five different regular and irregular zones. In our previous work we proved that analyzing the image with local attributes result in better retrieval efficiency and hence in this paper we computed the attributes by dividing the image into 64 regular and irregular zones. This experimentation carried out on IRMA 2008 and IRMA 2009 X-Ray image data sets. In this work we come up with some conclusions like wavelet based textural attributes, intensity features and Eigen values extracted from different regular zones worked well in retrieving the images over the features computed over irregular zones. We also determined like the set of image features in which form of zoning for different anatomical regions result in excellent retrieval of similar looking X-Ray images.
\end{abstract}

Keywords: Region Based Retrieval; Zoning Methods; Intensity attributes; Statistical attributes; SVD.

\section{Introduction}

Medical Image retrieval (MIR) is to organize digital medical image achieves by means of their visual content. The most common form of MIR is image search by its visual attributes. This mechanism deals with image classification, recognition and retrieval and these are the three important aspects of automatic image annotation. The annotation of medical image collections is an active research area. Annotation process is considered as concept detection where images pertaining to the same concept can be described linguistically in different ways based on the specific instance of the concept.

Integration into PACS is an essential step for the clinical use of a retrieval system. Medical images produced by various modalities are in DICOM format. Digital Imaging and Communication in medicine (DICOM) is a complex standard. It covers a broad range of imaging modalities and deal with both communications and storage of images [1].

According to the literature survey of [2] considerable amount of work had already done in medical image retrieval but still it is a challenging task for researchers. X-Ray images are very rich in texture and it is the highly considerable attribute for retrieval. Researchers are trying to explore other optimal features for best retrieval of $X$ - Ray images.

\section{Related Work}

Image retrieval based on 2-D region based visual features is presented. Region features obtained with thresholding. Object regions are extracted and structural features of each region described [3]. Optimization of high resolution content in $\mathrm{x}$ ray images with morphological opening and closing operations along with convolution and subtraction discussed in [4]. Paper [5, 6] discussed about texture classification and discrimination based on the energies of image subbands using DCT, wavelet and spatial partitioning. VisualSEEk is an image search engine that allows both color and spatial querying by using image regions and their colors, sizes, spatial location ships and relationships for retrieval. Able to perform automated extraction of localized regions and features, querying by both spatial and feature information, fast extraction from compressed data and developed fast indexing and retrieval techniques [7]. Images decomposed into blocks and wavelet coefficients are computed for each block and the query features compared with these features for fast retrieval of similar images from large databases [8].

ROI of the blood cell images is computed based on segmentation and shape, area, color and texture features computed in that region [9]. Global and semi local edge histograms generated from local histogram bins and compared for image matching [10]. Paper [11] developed a CBIR system in which user can select multiple regions of interest and can specify the relevance of their spatial layout in the retrieval process. Spectral graph theoretic frame work of normalized cuts is used in [12] to find the partitions of the image into regions of coherent texture and brightness. Translation invariant texture features provided by discrete wavelet coefficients in [13]. Statistical shape feature based image retrieval done in [14]. Image signature is calculated by finding gradient descriptor of texture features using a GLCM matrix by finding variance, energy, entropy, homogeneity, third order moment, inverse variance for image retrieval done in [15]. An efficient region based image re- 
trieval presented in [16] by applying a multistep approach to use in region based retrieval techniques in large databases. with this retrieval processing time got significantly reduced. In [17] images are represented as signatures based on the distribution of wavelet transform and weighted signature distances computed between query and database images and an adapted wavelet base is proposed. Medical image retrieval performed with energy efficient wavelet transforms such as Biorthogonal and Haar with Euclidean Distance as a similarity measure in [18]. Reference [19] listed all low level features as color, texture and shape of images to use in CBIR. A figure of merit called retrieval efficiency defined in [20]

\section{Methodology}

We experiment our work on IRMA 2008 database by choosing ten different classes of images: Abdomen, Spine, Lung, Skull, Breast, Hand, Shoulder, Feet, Ankle and Knee. 20\% of the database images are chosen as query set and the system is evaluated in terms of average precision for every image category and mean average precision (MAP) for all the 10 classes of images at $100 \%$ recall rate. Sample images of IRMA data set shown in fig 1.
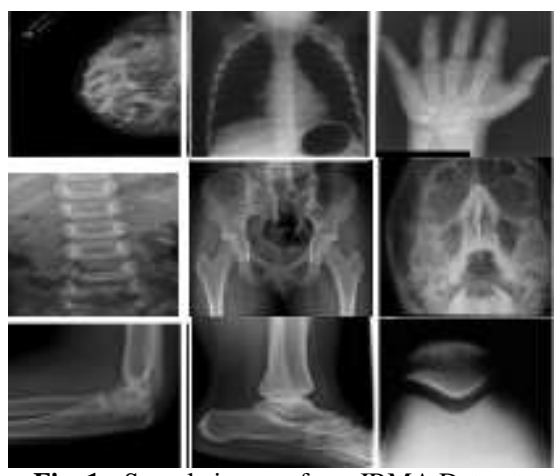

Fig. 1:. Sample images from IRMA Dataset

An empirical evaluation of our previous work illustrates that local features significantly improve retrieval performance when compared with global feature based retrieval.

Based on this evaluation, in the present work we divided the query and database images of $128 \times 128$ in pixel size into non overlapped 64 regular regions in sizes as $(16 \times 16)$ as squared blocks, $128 \times 2$ and $2 \times 128$ sized rectangular blocks as column zones and row zones respectively.

We also analyzed the system performance by partitioning the images into 64 non overlapped irregular sized regions as spiral zones and staircase zones. These five zone partitions shown in fig 2.a. to 2.e.
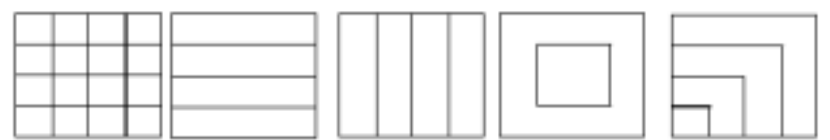

Fig 3.a:. Squared regions b. Row regions, c. Column regions d. Spiral regions e. Staircase regions

The proposed retrieval system is shown in fig 3 . The query image is partitioned into regular and irregular regions as shown in fig 2 . The data base images also divided into various non overlapping zones. Intensity attributes, texture attributes, statistical attributes transformed coefficients as DFT and DWT and Eigen values were determined for every zone of both query and database images and these features were used in finding the similar images.

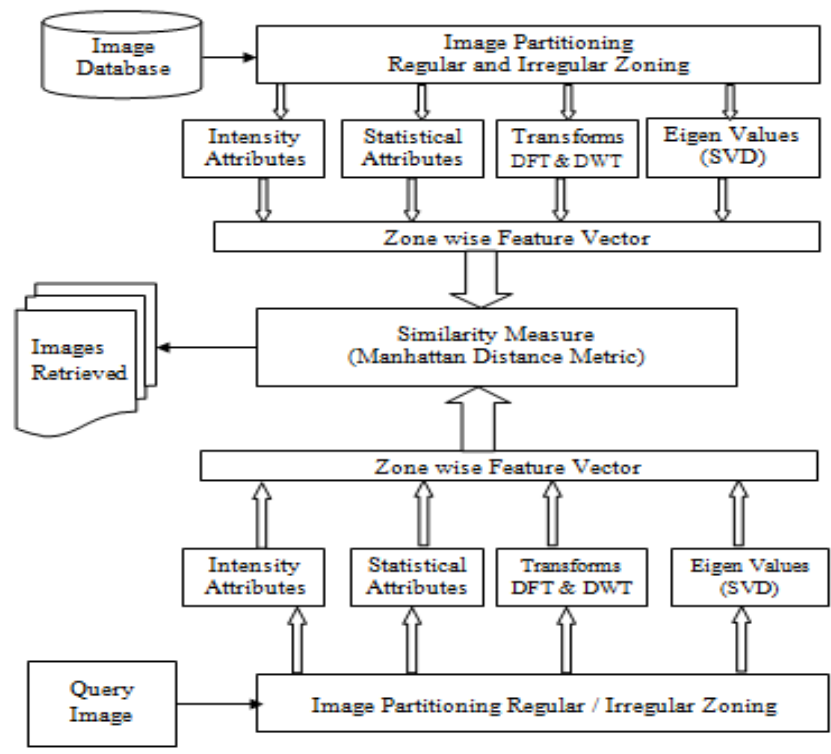

Fig.2:. Proposed Medical Image Retrieval System

\subsection{Intensity Attributes}

In query and database images sum of pixel values within the zone is computed by Eq.1. Probability of intensity distribution is determined for every zone by using the histogram approach as given in Eq. 2. These values computed for all the zones were used as intensity based feature vectors and are used to retrieve the similar looking images from database.

Sum of Pixels in a Zone $=\sum_{i} \sum_{j} f(i, j)$
Probability $f(i, j)=\frac{N(i, j)}{M}$

Where $f(i, j)$ denote the pixel values within the zone. $N(i, j)$ denote number of pixels having same gray value and $M$ represent total number of pixels within that zone.

\subsection{Statistical Coefficients}

Statistical features such as mean, standard deviation, variance, smoothness, uniformity, entropy and third order moments were calculated for every zone of the image. All these seven attributes as shown in eq. 3 to eq. 9 respectively, used as a feature vector for every individual zone of the image. All feature vectors of 64 zones of query and database images are checked with Manhattan distance for retrieval of similar looking images.

$$
\begin{aligned}
\operatorname{Mean}(\bar{g}) & =\sum_{g=0}^{L-1} g P(g) \\
& =\sum_{r} \sum_{r} \frac{I(r, c)}{M} \\
\text { Standard Deviation }\left(\sigma_{g}=\sqrt{\sum_{g=0}^{L-1}(g-\bar{g})^{2} P(g)}\right. & \\
\text { Variance } & =\sum_{g=0}^{L-1}(g-\bar{g})^{2} P(g) \\
\text { Smoothness } & =\frac{1}{1-\sum_{g=0}^{L-1}(g-\bar{g})^{2} P(g)}
\end{aligned}
$$




$$
\begin{aligned}
& \text { Entropy }=-\sum_{g=0}^{L-1} P(g) \log _{2}[P(g)] \\
& \text { Uniformity }=\sum_{g=0}^{L-1}[P(g)]^{2} \\
& \text { Third order Moment }=\frac{1}{\sigma_{g}^{3}} \sum_{g=0}^{L-1}(g-\bar{g})^{3} P(g)
\end{aligned}
$$

where $P(g)=\frac{N(g)}{M}$

\subsection{DWT and DFT Coefficients}

Every zone of query and database images is translated to frequency domain from spatial domain using Discrete Fourier Transform (DFT) and first order Discrete Wavelet Transform (DWT). DFT coefficients are used as a feature vector for similar image retrieval. LL band of a transformed image consists of maximum information about it and hence energy of LL band coefficients for every transformed region were used as a feature vector in retrieval process

\subsection{Eigen Values (SVD)}

By applying Singular Value Decomposition (SVD) for every zone of query and database images and principal diagonal values i.e. large Eigen values of the regions are used as feature sets for image retrieval.

\subsection{Similarity Measures}

The main issue in image retrieval systems is the number of dimensions of the feature vector which is normally huge. Manhattan distance metric measures the direct grid distance along the pixels and diagonal movements are not allowed. Manhattan distance metric given in Eq. 10 retrieve images at a faster rate when compared with Euclidean distance [21]. To speed up the retrieval process, we used this distance measure for all the five attributes computed from five zone partitions of the images

$$
\text { Manhatten Distance }=\sum_{i=1}^{n}\left|a_{i}-b_{i}\right|
$$

\subsection{Performance Metrics}

The presented retrieval system performance analyzed by retrieval efficiency in terms of average precision (AP) and Mean Average Precision (MAP) at 100\% recall rate as given in Eq.11, 12 and 13.

Average Precision $(A P)=$

Number of relevant images retrieved for a class of images

total number of images retrieved for a class of images

Mean Average Precision (MAP)

$=\frac{\text { No.ofrelevant images retrieved for all classes }}{\text { total number of images retrieved for all classes }}$

Recall Rate

$=\frac{\text { No. of relevant images retrieved }}{\text { Total Number of relevant images in the database }}$

\section{Results \& Discussions}

As presented in earlier section, we used 1000 images of IRMA 2008 and 1000 images of IRMA 2009 X - Ray image datasets. These images are categorized into 10 different classes of 200 images for each class. Each class represents the images of various anatomical regions viz. abdomen, spine, lungs, breast, skull, hand, shoulder, leg, knee and ankle. From every class $20 \%$ of images are chosen as query images and the retrieval efficiency is computed in terms of Average Precision (AP) for every class and Mean Average Precision for the entire data set with a recall rate of $100 \%$. i.e. the AP and MAP computed by retrieving the first 200 images (equal to the size of relevant images in the data set) that shown the least distance value with respect to query images.

The best retrieval efficiency with more than $75 \%$ of MAP obtained with features extracted from regular zones than features calculated from irregular zones. The comparison analysis of MAP for the features extracted from various zone partitions is shown in Table1. Graphical representation of comparison analysis is shown in fig 4 .

Table I: \% MAP for all queries
\begin{tabular}{|c|c|c|c|c|c|c|}
\hline ZONE & Sum & HG & Stat & DFT & DWT & SVD \\
\hline Square & 76 & 69 & 71 & 70 & 68 & 76 \\
\hline Row & 61 & 67 & 67 & 68 & 58 & 59 \\
\hline Column & 52 & 59 & 56 & 58 & 49 & 55 \\
\hline Staircase & 23 & 24 & 22 & 23 & 23 & 24 \\
\hline Spiral & 23 & 23 & 21 & 17 & 19 & 17 \\
\hline
\end{tabular}

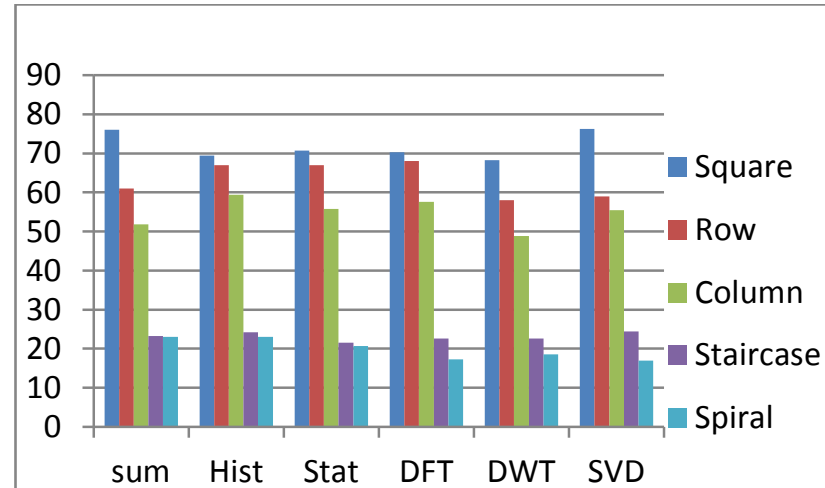

Fig.4:. Zone wise \% MAP comparison

From the comparison analysis we understood that squared and row wise regular regions out performed very well and hence we presented the class wise Average Precision (AP) for all the feature descriptors with square and row wise image partitioned zones in table 2 . From the table it is clearly understood that for abdomen, spine lungs, skull, knee and feet image classes regular zoning with sum and SVD features shown good retrieval efficiency and for breast, palm and shoulder classes row wise zoning with histogram and statistical features shown better retrieval over

\begin{tabular}{|c|c|c|c|c|c|c|c|c|c|c|c|}
\hline $\begin{array}{l}\text { Zo } \\
\text { nes }\end{array}$ & $\begin{array}{c}\text { Fea } \\
\text { ture } \\
\text { s }\end{array}$ & $\begin{array}{c}\text { ab- } \\
\text { dom } \\
\text { en }\end{array}$ & $\begin{array}{c}\text { sp } \\
\text { in } \\
\mathrm{e}\end{array}$ & $\begin{array}{c}\text { lu } \\
\text { ng } \\
\text { s }\end{array}$ & $\begin{array}{c}\text { sk } \\
\text { ul } \\
\text { l }\end{array}$ & $\begin{array}{l}\text { br } \\
\text { ea } \\
\text { st }\end{array}$ & $\begin{array}{c}\mathbf{k} \\
\mathbf{n} \\
\text { ee }\end{array}$ & $\begin{array}{l}\mathbf{f} \\
\mathbf{e} \\
\mathrm{e} \\
\mathrm{t}\end{array}$ & $\begin{array}{l}\text { p } \\
\text { al } \\
\text { m }\end{array}$ & $\begin{array}{c}\text { an } \\
\text { kl } \\
\text { e }\end{array}$ & $\begin{array}{l}\text { sho } \\
\text { uld } \\
\text { er }\end{array}$ \\
\hline \multirow{6}{*}{$\begin{array}{l}\text { sq } \\
\text { ua } \\
\text { re }\end{array}$} & $\begin{array}{c}\mathbf{S U} \\
\mathbf{M}\end{array}$ & 93 & 85 & 95 & $\begin{array}{l}7 \\
8\end{array}$ & 72 & $\begin{array}{l}7 \\
4\end{array}$ & $\begin{array}{l}7 \\
5\end{array}$ & 70 & 54 & 65 \\
\hline & HG & 90 & 82 & 88 & $\begin{array}{l}9 \\
1\end{array}$ & 54 & $\begin{array}{l}6 \\
4\end{array}$ & $\begin{array}{l}7 \\
2\end{array}$ & 62 & 37 & 54 \\
\hline & $\begin{array}{c}\text { ST } \\
\text { AT }\end{array}$ & 92 & 76 & 88 & $\begin{array}{l}8 \\
1\end{array}$ & 67 & $\begin{array}{l}6 \\
5\end{array}$ & $\begin{array}{l}6 \\
7\end{array}$ & 67 & 47 & 58 \\
\hline & $\begin{array}{c}\mathbf{D F} \\
\mathbf{T}\end{array}$ & 94 & 81 & 92 & $\begin{array}{l}6 \\
8\end{array}$ & 64 & $\begin{array}{l}7 \\
6\end{array}$ & $\begin{array}{l}5 \\
8\end{array}$ & 48 & 54 & 66 \\
\hline & $\begin{array}{c}\text { DW } \\
\mathbf{T}\end{array}$ & 94 & 81 & 92 & $\begin{array}{l}6 \\
8\end{array}$ & 64 & $\begin{array}{l}7 \\
6\end{array}$ & $\begin{array}{l}5 \\
8\end{array}$ & 48 & 54 & 66 \\
\hline & $\begin{array}{c}\text { SV } \\
\text { D }\end{array}$ & 94 & 86 & 96 & $\begin{array}{l}7 \\
3\end{array}$ & 72 & $\begin{array}{l}7 \\
4\end{array}$ & $\begin{array}{l}7 \\
4\end{array}$ & 72 & 55 & 65 \\
\hline ro & SU & 46 & 46 & 87 & 5 & 68 & 6 & 6 & 58 & 45 & 66 \\
\hline
\end{tabular}
square wise zoning. Optimal features with zoning methods for different anatomical X - Ray images shown in table 3 .

Table II: Class wise \% AP for squared and row wise regions 


\begin{tabular}{|c|c|c|c|c|c|c|c|c|c|c|c|}
\hline \multirow[t]{10}{*}{$\mathbf{w}$} & $\mathbf{M}$ & & & & 9 & & 5 & 9 & & & \\
\hline & HG & 72 & 73 & 84 & $\begin{array}{l}9 \\
1\end{array}$ & 57 & $\begin{array}{l}6 \\
6\end{array}$ & $\begin{array}{l}6 \\
6\end{array}$ & 70 & 40 & 56 \\
\hline & ST & & & & 7 & & 6 & 6 & & & \\
\hline & AT & 60 & 63 & 78 & 3 & 90 & 8 & 4 & 63 & 50 & 59 \\
\hline & DF & & & & 6 & & 6 & 5 & & & \\
\hline & $\mathbf{T}$ & 89 & 74 & 81 & 4 & 67 & 7 & 8 & 53 & 53 & 72 \\
\hline & DW & & & & 5 & & 5 & 7 & & & \\
\hline & $\mathbf{T}$ & 45 & 47 & 79 & 7 & 60 & 7 & 3 & 57 & 45 & 54 \\
\hline & SV & & & & 6 & & 6 & 6 & & & \\
\hline & D & 46 & 52 & 86 & 1 & 56 & 1 & 9 & 58 & 48 & 56 \\
\hline
\end{tabular}

Table III: Optimal feature set for various classes X-Ray images with Zoning Techniques

\begin{tabular}{|c|c|c|c|c|c|c|c|c|c|c|}
\hline Zone & $\begin{array}{c}\text { ab- } \\
\text { dom } \\
\text { en }\end{array}$ & $\begin{array}{c}\text { spi } \\
\text { ne }\end{array}$ & $\begin{array}{c}\text { lu } \\
\text { ng } \\
\text { S }\end{array}$ & $\begin{array}{l}\text { sk } \\
\text { ull }\end{array}$ & $\begin{array}{l}\text { bre } \\
\text { ast }\end{array}$ & $\begin{array}{l}\text { kn } \\
\text { ee }\end{array}$ & $\begin{array}{l}\text { fe } \\
\text { et }\end{array}$ & $\begin{array}{l}\text { pa } \\
\text { lm }\end{array}$ & $\begin{array}{l}\text { an } \\
\text { kle }\end{array}$ & $\begin{array}{l}\text { shou } \\
\text { lder }\end{array}$ \\
\hline $\begin{array}{l}\text { SQU } \\
\text { ARE }\end{array}$ & $\begin{array}{c}\text { SVD } \\
\text { DW } \\
\text { T, } \\
\text { DFT }\end{array}$ & $\begin{array}{c}\mathrm{S} \\
\mathrm{V} \\
\mathrm{D}, \\
\mathrm{S} \\
\mathrm{U} \\
\mathrm{M}\end{array}$ & $\begin{array}{c}\mathrm{S} \\
\mathrm{V} \\
\mathrm{D}, \\
\mathrm{S} \\
\mathrm{U} \\
\mathrm{M}\end{array}$ & $\begin{array}{l}\mathrm{H} \\
\mathrm{G}\end{array}$ & - & $\begin{array}{c}\text { D } \\
\text { FT } \\
\text {, } \\
\text { D } \\
\text { W } \\
\text { T }\end{array}$ & $\begin{array}{l}\text { S } \\
\text { V } \\
\text { D }\end{array}$ & $\begin{array}{c}\mathrm{S} \\
\mathrm{V} \\
\mathrm{D}, \\
\mathrm{S} \\
\mathrm{U} \\
\mathrm{M}\end{array}$ & $\begin{array}{c}\text { SV } \\
\text { D }\end{array}$ & $\begin{array}{c}\text { DFT } \\
\text { DW } \\
\text { T }\end{array}$ \\
\hline $\begin{array}{c}\% \\
\text { MAP }\end{array}$ & 94 & 86 & 96 & 91 & - & 76 & 75 & 72 & 55 & 66 \\
\hline $\begin{array}{c}\text { RO } \\
\text { W }\end{array}$ & - & - & - & $\begin{array}{l}\mathrm{H} \\
\mathrm{G}\end{array}$ & $\begin{array}{l}\text { ST } \\
\text { AT }\end{array}$ & - & - & $\begin{array}{l}\mathrm{H} \\
\mathrm{G}\end{array}$ & $\begin{array}{c}\text { DF } \\
T\end{array}$ & $\begin{array}{l}\text { SU } \\
\text { M }\end{array}$ \\
\hline $\begin{array}{c}\% \\
\text { MAP }\end{array}$ & - & - & - & 91 & 90 & - & - & 70 & 53 & 66 \\
\hline
\end{tabular}

\section{Conclusion}

In this paper we presented our work on comparative analysis for retrieval of $2000 \mathrm{X}$ - ray images of ten different anatomical regions with 200 images of each class. The proposed retrieval system checked with $20 \%$ of query images from each class. The images used are used from IRMA 2008 and IRMA 2009 datasets. The image attributes were computed by partitioning the images into 64 zones of three regular sized (square, row and column) and two irregular sized (spiral and staircase) regions. The image attributes include sum of pixel values, probability distributions, statistical features, transformed coefficients DFT and DWT and Eigen values zone wise. All these feature vectors were compared with Manhattan distance for similarity check between query and database images. Through the experimentation we learnt that Eigen values computed with SVD over square partitioned zones result in $75 \%$ to $95 \%$ Average Precision for abdomen, spine, lungs, knee, palm and ankle classes and histogram features of squared and row regions for skull images with $91 \%$, statistical features computed over row regions result in $90 \%$ average precision for breast images and about $75 \%$ average precision by row regions computed DWT and DFT for feet and shoulder images respectively.

\section{Acknowledgement}

We would like to thank Thomas M. Lehmann, RWTH Achen University, Germany, for providing IRMA 2008 and IRMA 2009 datasets for our experimentatal work

\section{References}

[1] Baljon, M. H., and M. G. J. M. Gerritsen. "Holland goes DICOM." Computers in Cardiology 1995. IEEE 1995.

[2] Nalini, Mrs P., and B. L. Malleswari. "Review on Content Based Image Retrieval: From Its Origin to the New Age." International Journal of Research Studies in Science, Engineering and Technology (IJRSSET) Vol 3. Issue 2, Feb 2016,PP 18-41. ISSN: 2349-4751

[3] Shyu, Chi-Ren, C. E. Brodley, A. C. Kak, Akio Kosaka, A Aisen, and L. Broderick. "Local versus global features for content-based image retrieval." In Content-Based Access of Image and Video Libraries, 1998. Proceedings. IEEE Workshop on, pp. 30-34. IEEE, 1998.

[4] Yamamoto, Akio, and Mikio Takagi. "Extraction of object features from image and its application to image retrieval." Pattern Recognition, 1988., 9th International Conference on. IEEE, 1988.

[5] Johnson, Roger H., et al. "Optimal information retrieval from complex low frequency backgrounds in medical images." Engineering in Medicine and Biology Society, 1989. Images of the Twenty-First Century., Proceedings of the Annual International Conference of the IEEE Engineering in. IEEE, 1989.

[6] Smith, John R., and Shih-Fu Chang. "Transform features for texture classification and discrimination in large image databases." Image Processing, 1994. Proceedings. ICIP-94., IEEE International Conference. Vol. 3. IEEE, 1994.

[7] Ma, Wei-Ying, and B. S. Manjunath. "A comparison of wavelet transform features for texture image annotation." icip. IEEE, 1995.

[8] Smith, John R., and Shih-Fu Chang. "VisualSEEk: a fully automated content-based image query system." Proceedings of the fourth ACM international conference on Multimedia. ACM, 1997.

[9] Remias, Edward, et al. "Supporting content-based retrieval in large image database systems." Multimedia Database Management Systems. Springer US, 1997. 61-78.

[10] Comaniciu, Dorin, Peter Meer, David Foran, and Attila Medl. "Bimodal system for interactive indexing and retrieval of pathology images." InApplications of Computer Vision, 1998. WACV'98. Proceedings., Fourth IEEE Workshop on, pp. 76-81. IEEE, 1998.

[11] Park, Dong Kwon, Yoon Seok Jeon, and Chee Sun Won. "Efficient use of local edge histogram descriptor." In Proceedings of the 2000 ACM workshops on Multimedia, pp. 51-54. ACM 2000.

[12] Moghaddam, Baback, Henning Biermann, and Dimitris Margaritis. "Regions-of-interest and spatial layout for contentbased image retrieval." Multimedia Tools and Applications 14, no. 2 (2001): 201-210.

[13] Malik, Jitendra, Serge Belongie, Thomas Leung, and Jianbo Shi "Contour and texture analysis for image segmentation." International journal of computer vision 43, no. 1 (2001): 7-27.

[14] Özden, Mustafa, and Ediz Polat. "Image segmentation using color and texture features." In Signal Processing Conference, 2005 13th European, pp. 1-4. IEEE, 2005.

[15] Brandt, Sami, Jorma Laaksonen, and Erkki Oja. "Statistical shape features for content-based image retrieval." Journal of Mathematical Imaging and Vision 17, no. 2 (2002): 187-198.

[16] Felipe, Joaquim Cezar, Agma JM Traina, and C. Traina. "Retrieval by content of medical images using texture for tissue identification." In Computer-Based Medical Systems, 2003. Proceedings. 16th IEEE Symposium, pp. 175-180. IEEE, 2003.

[17] Weber, Roger, and Michael Mlivoncic. "Efficient region-based image retrieval." In Proceedings of the twelfth international conference on Information and knowledge management, pp. 69-76. ACM, 2003.

[18] Unay, Devrim, and Ahmet Ekin. "Intensity versus texture for medical image search and retrieval." In 2008 5th IEEE International Symposium on Biomedical Imaging: From Nano to Macro, pp. 241-244. IEEE, 2008

[19] Rajakumar, K., and S. Muttan. "Medical image retrieval using energy efficient wavelet transform." In Computing Communication and Networking Technologies (ICCCNT), 2010 International Conference on, pp. 1-5. IEEE, 2010.

[20] Hussain, Chesti Altaff, D. Venkata Rao, and S. Aruna Mastani "Low level feature extraction methods for Content Based Image Retrieval." In Electrical, Electronics, Signals, Communication and Optimization (EESCO), 2015 International Conference on, pp. 1-5. IEEE, 2015.

[21] Pasumarthi, N.; Malleswari, L.: 'An empirical study and comparative analysis of content based image retrieval (CBIR) techniques with various similarity measures', IET Conference Proceedings, 2016, p. -, DOI: 10.1049/cp.2016.1529 IET Digital Library, http://digitalbrary.theiet.org/content/conferences/10.1049/cp.2016.1529 